\title{
RECONOCIMIENTO IDENTITARIO Y ACADEMICISMO EN EL CURRÍCULUM MUSICAL DE EDUCACIÓN PRIMARIA
}

\author{
Pablo Marín-Liébana \\ José Salvador Blasco Magraner \\ Ana María Botella Nicolás \\ Universidad de Valencia
}

\begin{abstract}
RESUMEN: Durante los últimos años ha crecido el interés por el reconocimiento de las identidades musicales de los estudiantes en el sistema educativo. Diferentes desarrollos teóricos críticos sostienen la necesidad de darles voz e incluir sus preferencias musicales en los distintos niveles curriculares. El objetivo de este trabajo es analizar la representación de las identidades y preferencias musicales de los estudiantes en el currículum oficial de educación musical primaria durante las últimas tres décadas. Para ello, se ha utilizado el análisis de contenidos con el fin de estudiar el repertorio prescrito durante las tres reformas que han tenido lugar desde 1990. Se ha encontrado que el currículum presenta referencias directas e indirectas al repertorio. Las primeras están formadas principalmente por música folklóricotradicional $y$, en la actualidad, también por académica, aunque la mayor parte son referencias indeterminadas que dejan flexibilidad de elección a los docentes. En cuanto a las referencias indirectas, estas favorecen la música académica y su sistema de aprendizaje formal. Por su parte, las preferencias e identidades de los estudiantes son prácticamente inexistentes. Por tanto, se concluye que el currículum musical estudiado tiene un carácter academicista y que no refleja las propuestas de la educación musical crítica.
\end{abstract}

PALABRAS CLAVE: educación musical, pedagogía crítica, educación primaria, política educativa, currículum.

\section{IDENTITY RECOGNITION AND ACADEMICISM IN THE MUSIC CURRICULUM OF PRIMARY EDUCATION}

\footnotetext{
ABSTRACT: In recent years, interest in the recognition of the musical identities of students in the educational system has grown. Different critical theories support the need to give them a voice and include their musical preferences at different curricular levels. In this sense, the objective of this
} 
work is to analyze the representation of the students' musical identities and preferences in the official curriculum of primary music education during the last three decades. For this, a content analysis methodology has been used, in order to study the repertoire prescribed during the three reforms that have taken place since 1990. It has been found that the curriculum presents direct or indirect references to the repertoire. The former are mainly made up of folk-traditional music and currently also academic, although most of them are indeterminate references that leave flexibility of choice to teachers. Within the indirect references, they favor academic music and its formal learning system. Besides, students' preferences and identities are practically non-existent. Therefore, it is concluded that the musical curriculum studied is academiccentered and does not reflect the proposals of critical musical education.

KEYWORDS: Music education, critical pedagogy, primary education, educational policy, curriculum.

Recibido: 21/09/2020

Aceptado: 25/02/2021

Correspondencia: Pablo Marín-Liébana, Facultad de Magisterio, Universidad de Valencia, Avenida Tarongers, 4, 46022 Valencia. Email: pablo.marin-liebana@uv.es

\section{INTRODUCCIÓN}

El interés por el estudio de las identidades musicales en la escuela ha crecido recientemente como consecuencia de dos cuestiones. Por un lado, la crisis de la creencia en los valores universales y el cambio hacia la contextualidad, el situacionismo y la deconstrucción de las estructuras de poder. Por el otro, la transición de un modelo que transmite conocimiento a otro que otorga el protagonismo al aprendizaje relacional y a las prácticas compartidas dentro de comunidades sociales (Westerlund, Partti y Karlslen, 2017). Este doble giro, que sitúa el foco en la potencialidad educativa de las experiencias concretas de los individuos, encuentra eco en diversos desarrollos teóricos. De una parte, tanto la tradición de la pedagogía crítica como la vinculada con la justicia social y la diversidad, postulan la lucha por el reconocimiento de la identidad cultural frente a las políticas de dominación y estandarización (Apple, Au y Gandin, 2009; Gay, 2000; Pugach, Gomez-Najarro y Matewos, 2018).

Por otra parte, dentro de los marcos conceptuales de la educación para la democracia (Wright, 2009) y de la propia pedagogía crítica para la educación musical (Abrahams, 2007; Gowan, 2016) cobra importancia la voz de los estudiantes y su participación en los distintos espacios sociales (Giroux, 1989; Laurence, 2009). Así, estas tradiciones sostienen tanto la necesidad de reconocer y dar valor a sus aprendizajes y vivencias (Rimmer, 2013), como de consultarles, establecer un diálogo sobre su propio aprendizaje y empoderarles en dicho proceso (Flutter y Rudduck, 2004). En definitiva, se trata de dejar de pensarlos como personas en construcción para pensarlos como individuos de pleno derecho con una visión propia del mundo, unas necesidades y unos 
deseos (Catling, 2014). Dentro de la educación musical, reconocer sus identidades implica conocer sus procesos de enculturación y preferencias musicales e incorporarlos en las diferentes dimensiones curriculares (Green, 2004; Laurence, 2009).

Este trabajo tiene como objetivo conocer el repertorio musical prescrito en la política curricular española de las últimas décadas, así como el grado de representación de las identidades musicales de los estudiantes en el mismo. Para ello, se han analizado los decretos de desarrollo curricular de la educación primaria en la Comunidad Valencia durante los últimos 30 años, correspondientes al periodo democrático actual.

\section{MarCo teórico}

Este trabajo se fundamenta en dos ejes teóricos. Por un lado, en el reconocimiento de las identidades musicales de los estudiantes a partir de la incorporación de sus preferencias musicales en las aulas de música. Por el otro, en el papel hegemónico que ejerce la música académica occidental en el currículum musical y el efecto que esto produce en dicha incorporación.

\section{Las identidades musicales de los estudiantes en la educación primaria}

La identidad es un concepto complejo que ha sido abordado desde diferentes perspectivas y análisis, resistiéndose a una definición monolítica y ampliamente aceptada (Marín-Liébana et al., 2020a). En el ámbito musical, Green (2011) formula una definición integradora en la que incluye la diversidad de experiencias que la constituyen y abarca preferencias musicales, valores, prácticas, habilidades y conocimientos. Por otra parte, Hargreaves, MacDonald y Miell (2017) diferencian entre las identidades en la música (IEM) y la música en las identidades (MEI). Las primeras están formadas por roles social y culturalmente establecidos dentro de la música, como compositor, intérprete, docente, crítico o consumidor/público/fan, y por categorías conceptuales relacionadas con instrumentos, como viento madera, cuerda frotada o percusión, o con géneros musicales, como pop, rock, jazz o música clásica. Las segundas están constituidas por las formas en que la música desarrolla diferentes aspectos de nuestra identidad general, como pueden ser las identidades vinculadas con el género, la edad, las nacionalidades, la discapacidad o la propia personalidad. Dado que, en el marco de la educación musical general, la mayoría de estudiantes no tienen formación específica, sus identidades musicales están principalmente determinadas por sus roles de consumidor/público/fan, sus preferencias musicales y su vinculación a géneros musicales.

Desde un punto de vista evolutivo, las identidades son especialmente relevantes en la etapa de la adolescencia, momento en el que la música juega un papel fundamental en cuestiones como la construcción de su sexualidad, los roles de género o la ideología política (Kelly, Pomerantz y Currie, 2005; Künzler, 2011; Larson y Kubey, 1983; Westerlund et al., 2017). En esta etapa, las preferencias musicales de los individuos están formadas fundamentalmente por la música popular urbana (MPU) (Lamont, Hargreaves, Marshall y Tarrant, 2003; Marín-Liébana y Botella, 2020; Megías y Rodríguez, 2003), inclinación que comienza a aparecer alrededor de los 9 años (Grembis y Schellberg, 2003; Hargreaves, North y Tarrant, 2010). Por tanto, durante la segunda mitad de la etapa de educación primaria, especialmente a partir de $5^{\circ}$ 
curso, momento en el que los estudiantes entran en la adolescencia temprana, sus preferencias e identidades musicales se relacionan con dicho repertorio.

\section{La hegemonía cultural de la música académica occidental}

Diversos estudios apuntan la existencia de una hegemonía de la música académica occidental en las políticas curriculares de la educación musical a lo largo de todo el mundo. Por un lado, en algunos países asiáticos (Ishii, 2018; Lum y Dairianathan, 2014), africanos (Barghi, Zakaria, Hamzah, y Hashim, 2017; Bresler, 2000) y sudamericanos (Lucas et al., 2016; Poblete, 2010), este repertorio fue impuesto durante los procesos colonizadores, si bien desde las últimas décadas del siglo XX hay una tendencia crítica hacia la validación de las manifestaciones musicales locales y la construcción de un currículum multicultural. Por otro lado, en países como Estados Unidos, Canadá, Australia, Reino Unido, España o Chipre, la reducción del repertorio académico occidental tiene como objetivo el reconocimiento de la pluralidad musical en un mundo globalizado y la ampliación de las experiencias y conocimientos musicales de los estudiantes (Monk y Poston, 1999). Sin embargo, a pesar transitar hacia la prescripción de un currículum multicultural desde el punto de vista del repertorio, los modos de enseñanza, evaluación y conceptualización siguen estando vinculados predominantemente al repertorio occidental hegemónico, por lo que todavía existe una relación de subordinación del resto de manifestaciones musicales con respecto a la tradición académica occidental (Cain, 2015b; Economidou, 2006; González, 2018; Hess, 2015).

En este sentido, en relación con la música popular urbana, que constituye las preferencias e identidades de los estudiantes de los últimos cursos de la educación musical primaria, algunos autores señalan que no es suficiente incorporarla como repertorio, ya que se siguen utilizando las formas tradicionales de enseñanza-aprendizaje de la música académica occidental (Green, 2004, 2006; Gurgel, 2019; Snell, 2009). Así, sostienen que también hay que añadir las estrategias propias del repertorio, conocidas con el nombre de aprendizaje informal (Green, 2005, 2006; Woody, 2007; Wright, 2008). Diversos trabajos han señalado las características principales que tendría una metodología educativa basada en dicho aprendizaje informal (Chua y Ho, 2017; Davis, 2013; Green, 2004, 2006; Snell, 2009; Wright, 2008). De forma sintética, los estudiantes deberían poder elegir la música que quieren trabajar en el aula, lo que implicaría utilizar piezas conocidas por estos que configurasen sus preferencias; se desarrollaría la creatividad, integrando la escucha con procesos de improvisación y composición, y el pensamiento crítico; se fomentaría un aprendizaje más horizontal basado en la colaboración dentro de un grupo de iguales; se sustituiría la notación tradicional por las grabaciones como forma de acceso a la música, lo que implicaría aprender de oído; no se estructuraría el aprendizaje desde lo simple a lo complejo, sino que se dejaría que los estudiantes marcasen su propio camino; y se utilizarían las tecnologías electrónicas y digitales relacionadas con la música popular urbana.

\section{Método}

Para alcanzar el objetivo planteado, se ha utilizado una metodología de análisis de contenido mixto que combina la descripción cuantitativa de frecuencias y proporciones con la interpretación cualitativa de textos. Este método, cuya finalidad 
es el estudio de documentos y el establecimiento de inferencias con respecto a sus contextos de uso, está caracterizado por implementar procedimientos empíricos y sistemáticos.

\section{MUeSTRA}

Las unidades de muestreo utilizadas han sido los tres decretos autonómicos promulgados por el Consell valenciano dentro del período democrático, que abarca desde finales de la década de 1970 hasta la actualidad, en los que se establece el currículum de la educación primaria en la Comunidad Valenciana (tabla 1). Para facilitar el análisis, a cada decreto se le ha asignado un código en relación con el año en el que fue publicado en el Diari Oficial de la Comunitat Valenciana.

Tabla 1. Unidades de muestreo

\begin{tabular}{cc}
\hline Código & DeCreto \\
\hline D1992 & $\begin{array}{r}\text { Decreto 20/1992, de } 17 \text { de febrero, del Gobierno Valenciano, por el que se esta- } \\
\text { blece el currículo de la Educación Primaria en la Comunidad Valenciana }\end{array}$ \\
\hline D2007 & $\begin{array}{c}\text { Decreto } 111 / 2007, \text { de } 20 \text { de julio, del Consell, por el que se establece el currí- } \\
\text { culo de la Educación Primaria en la Comunitat Valenciana }\end{array}$ \\
\hline D2014 & $\begin{array}{c}\text { Decreto 108/2014, de } 4 \text { de julio, del Consell, por el que establece el currículo } \\
\text { y desarrolla la ordenación general de la educación primaria en la Comunitat } \\
\text { Valenciana }\end{array}$ \\
\hline
\end{tabular}

\section{Instrumento de medida}

Se ha empleado un sistema de categorías de análisis que ha sido elaborado a partir de los conceptos presentados en el marco teórico y los tipos de formulaciones sobre el repertorio que aparecen en los documentos. De esta manera, se ha diferenciado inicialmente entre referencias directas al repertorio (RDR), que prescriben cuestiones como el estilo o la vinculación cultural, y referencias indirectas al repertorio (RIR), que lo hacen en relación con los tipos de instrumentos, agrupaciones y sistemas de aprendizaje vinculados a una tradición musical concreta. Dentro las RDR, se ha establecido una categorización que separa las determinadas y no determinadas (tabla 2).

Tabla 2. Categorías de análisis dentro de las $R D R$

\begin{tabular}{ccc}
\hline Categorías & Subcategorías & Ejemplos \\
\hline & & $\begin{array}{c}\text { Piezas musicales, obras vocales, música instrumental, } \\
\text { fragmentos, ejemplos musicales }\end{array}$ \\
No Determinado & $\begin{array}{c}\text { De distintas características, de diferentes tipos, variadas } \\
\text { De distintas/diferentes culturas }\end{array}$ \\
& De distintos/diversos/diferentes estilos, de diferentes épo- \\
cas/géneros & Canciones, retahílas, danzas, cánones \\
\hline
\end{tabular}




\begin{tabular}{ccc}
\hline Categorías & Subcategorías & Ejemplos \\
\hline & $\begin{array}{c}\text { Dimensión } \\
\text { cultural deter- } \\
\text { minada } \\
\text { Determinado }\end{array}$ & $\begin{array}{c}\text { De la Comunitat Valenciana, valencianas, españolas, de } \\
\text { las culturas mediterráneas, del mundo }\end{array}$ \\
& $\begin{array}{c}\text { Género, esti- } \\
\text { lo o período } \\
\text { determinado } \\
\text { (GEPD) }\end{array}$ & $\begin{array}{c}\text { Infantil, tradicional, popular, didáctica, clásica, de los } \\
\text { siglos XVII y XVIII/XVI al XIX/XV al XX, de la época con- } \\
\text { temporánea, contemporánea, de actualidad, del folklore, } \\
\text { de otras épocas, flamenco, jazz }\end{array}$ \\
\hline
\end{tabular}

En cuanto a las RIR, se han analizado los elementos curriculares en función de las categorías música académica (MA), música no académica (MNA), aprendizaje formal (AF) y aprendizaje informal (AI). Del mismo modo que con las RDR, a cada una de estas categorías se le ha asignado una serie de subcategorías que engloban un conjunto de formulaciones curriculares concretas, tal y como se muestra en la tabla 3. Estas etiquetas no son unívocas ni estrictamente exclusivas, sino que se han seleccionado por pertenecer en mayor medida a una tradición musical específica. Es decir, a pesar de no constituir subcategorías ontológicamente necesarias relacionadas con las categorías en las que se engloban, sí que forman parte de sus tradiciones de uso contingente.

Tabla 3. Categorías de análisis dentro de las RIR

\begin{tabular}{|c|c|c|}
\hline Categorías & SUBCATEGoRíAS & EJEMPLOS \\
\hline \multirow{5}{*}{$\begin{array}{c}\text { MúSICA } \\
\text { ACADÉMICA } \\
\text { (MA) }\end{array}$} & $\begin{array}{l}\text { Instrumentos } \\
\text { académicos }\end{array}$ & $\begin{array}{l}\text { Familias orquestales, familias instrumentales (cuer- } \\
\text { da, viento metal, viento madera y percusión) }\end{array}$ \\
\hline & $\begin{array}{l}\text { Agrupaciones } \\
\text { académicas }\end{array}$ & Orquesta, solo, dúo, trío, cuarteto, coro y orquesta \\
\hline & $\begin{array}{l}\text { Figura de la } \\
\text { dirección }\end{array}$ & Respetar a la persona que asume la dirección \\
\hline & Obra & Obra \\
\hline & Audición silenciosa & $\begin{array}{l}\text { Valoración del silencio como elemento indispen- } \\
\text { sable durante la audición musical, actitud atenta y } \\
\text { silenciosa durante la audición musical }\end{array}$ \\
\hline \multirow{2}{*}{$\begin{array}{l}\text { MÚSICA NO } \\
\text { ACADÉMICA } \\
\text { (MNA) }\end{array}$} & $\begin{array}{l}\text { Instrumentos no } \\
\text { académicos }\end{array}$ & $\begin{array}{c}\text { Instrumentos del folklore, de la MPU, electrónicos, } \\
\text { escolares, del aula, Orff, realizados con materiales } \\
\text { escolares }\end{array}$ \\
\hline & $\begin{array}{c}\text { Agrupaciones no } \\
\text { académicas }\end{array}$ & Rondallas \\
\hline \multirow[t]{2}{*}{$\begin{array}{l}\text { APRENDIZAJE } \\
\text { FORMAL } \\
\text { (AF) }\end{array}$} & $\begin{array}{l}\text { Teoría/análisis } \\
\text { musical }\end{array}$ & $\begin{array}{c}\text { Matices dinámicos y agógicos, intervalos, acordes, } \\
\text { escalas, forma, textura, tipos de voces (soprano, } \\
\text { alto, tenor y bajo), tonalidad, tempo (allegro, andan- } \\
\text { te, adagio, vivace, largo) }\end{array}$ \\
\hline & Notación escrita & Lectura de pentagramas, claves, compases, figuras \\
\hline
\end{tabular}




\begin{tabular}{ccc}
\hline CATEGORÍAs & SubCATEGorías & Ejemplos \\
\hline $\begin{array}{c}\text { APRENDIZAJE } \\
\text { FORMAL } \\
(\mathrm{AF})\end{array}$ & $\begin{array}{c}\text { Perfeccionamiento } \\
\text { técnico }\end{array}$ & $\begin{array}{c}\text { Buscando la precisión, cuidando el fraseo y la } \\
\text { articulación, perfeccionamiento y precisión en la } \\
\text { técnica instrumental, práctica de técnicas básicas, } \\
\text { ejercicios } \\
\text { Interpretación }\end{array}$ \\
\hline $\begin{array}{c}\text { APRENDIZAJE } \\
\text { INFORMAL } \\
(\mathrm{Al})\end{array}$ & Composición & Creación, invención, composición \\
& Improvisación & Improvisación \\
\hline
\end{tabular}

\section{Procedimiento}

Se ha seguido el procedimiento propuesto por Krippendorff (2013), el cual consta de seis fases: unitarización, muestreo, codificación, representación, inferencia y narración. En primer lugar, se establecieron las unidades de muestreo y las de registro, siendo las primeras los tres decretos señalados y las segundas sus elementos curriculares (EC). Tras una primera aproximación al análisis, se diseñó el sistema de codificación, combinando categorías emergentes con apriorismos teóricos extraídos de la literatura científica, y se codificó cada una de las unidades de registro. Seguidamente, las diferentes categorías y subcategorías de análisis fueron analizadas y representadas gráficamente en base a la frecuencia de aparición en las unidades de muestreo. Por último, los resultados fueron complementados con un análisis cualitativo, se desprendieron una serie de inferencias sobre la práctica educativa y se construyó una narración que facilitara su difusión.

\section{Análisis de datos}

En el análisis cuantitativo, se han utilizado como unidades de registro tanto los elementos curriculares (EC), formados por contenidos y criterios de evaluación, como las referencias al repertorio $(R R)$, consistentes en cualquier formulación que, de forma directa o indirecta, haga referencia a un tipo de música específico. De esta forma, en un EC puede haber ninguna, una o más de una RR. Estas unidades se han codificado en base al sistema de categorización establecido y se han analizado mediante la descripción estadística del conteo de proporciones. En cuanto al estudio cualitativo, se ha realizado un análisis interpretativo de los elementos textuales que contextualizan los EC, es decir, de las introducciones, los objetivos y la estructura del currículum. Así, esta aproximación hermenéutica complementa y contribuye a la comprensión de los resultados encontrados.

\section{ANÁLISIS DE RESULTADOS}

Los resultados obtenidos se exponen en tres apartados: análisis cuantitativo de las RDR, análisis cuantitativo de las RIR y análisis cualitativo. 


\section{Análisis cuantitativo de las RDR}

El análisis de las RDR se muestra en dos niveles diferenciados: su nivel de determinación y su distribución interna. Con respecto al primero, se ha observado una relación inversa entre el nivel de determinación y la cantidad de EC (figura 1). Además, los porcentajes son relativamente similares en los tres decretos, oscilando entre el $17.86 \%$ y el $24.53 \%$ en el nivel determinado, y entre el $85.71 \%$ y el $98.57 \%$ en el no determinado. En cuanto a las subcategorías, ambas presentan un patrón de crecimiento en D2007 y decrecimiento en D2014 (figura 2).

Figura 1. Nivel de determinación de las $R D R$

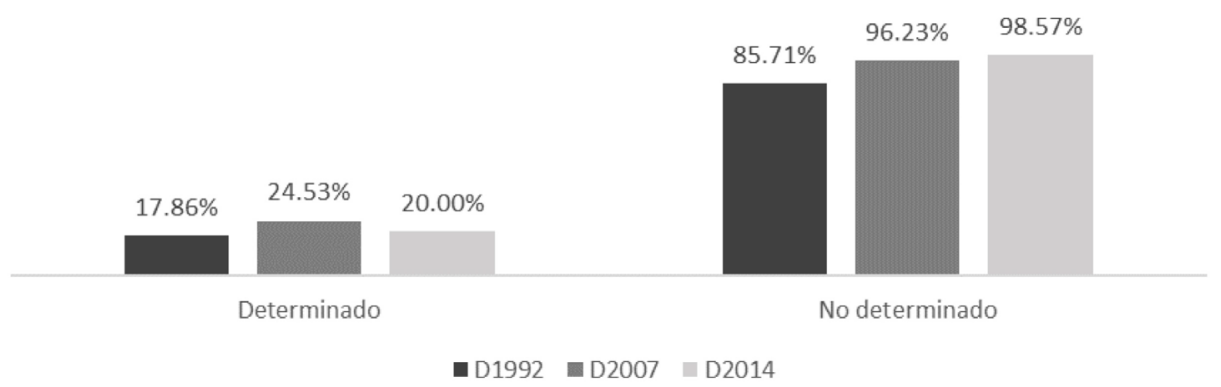

Figura 2. Distribución de las $R D R$

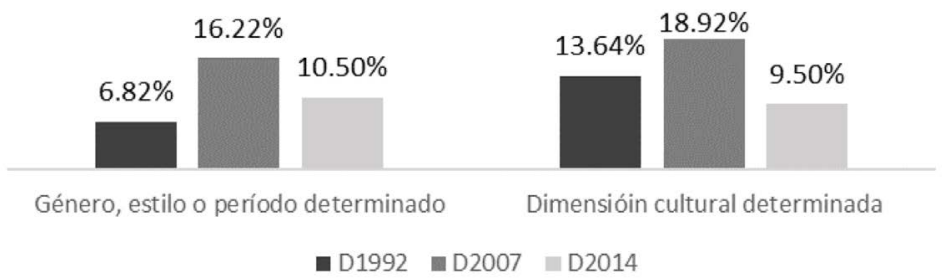

En relación con la distribución de las RDR sobre el GEPD, se observa que el D1992 solo hace alusiones a la música folklórica y tradicional, mientras que a partir de D2007 aparece también la música infantil, la académica, la popular y la didáctica (figura 3). En dicho decreto, llama la atención tanto el protagonismo de la primera como el hecho de ser el único en el que aparece el término popular. Por otro lado, el decreto D2014 supone la aparición de la música actual y la referencia a otros géneros distintos a los académico-occidentales. Asimismo, el decreto D2007 es el único que prescribe de forma indeterminada épocas distintas a la actual (figura 4). 
Figura 3. Distribución de las RDR relativas al GEPD (I)

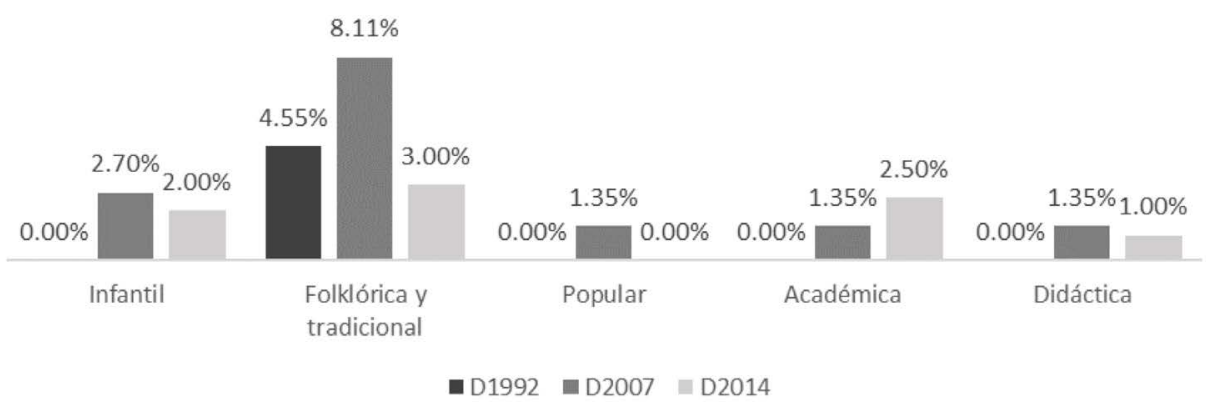

Figura 4. Distribución de las RDR relativas al GEPD (II)

\begin{tabular}{|c|c|c|c|c|c|}
\hline \multicolumn{2}{|c|}{$1.35 \%$} & & \multicolumn{3}{|c|}{$2.27 \%$} \\
\hline $0.00 \%$ & $0.00 \%$ & $0.00 \% 0.00 \%{ }^{0.50 \%}$ & $0.00 \% 0.00 \%{ }^{0.50 \%}$ & $0.00 \%$ & $1.00 \%$ \\
\hline \multirow{2}{*}{\multicolumn{2}{|c|}{ Otras épocas }} & Otros géneros & De actualidad & Contempor: & nea \\
\hline & & — D1992 & $007=D 2014$ & & \\
\hline
\end{tabular}

\section{Análisis cuantitativo de las RIR}

El análisis de las RIR se muestra en dos niveles diferenciados: en primer lugar, la proporción que se relaciona con la formalidad del aprendizaje y el academicismo del repertorio y, en segundo lugar, la distribución interna de cada una de ellas. Así, al comparar los tres decretos, se observa un esquema similar, de forma que el AF es el más frecuente, situándose entre el $31.02 \%$ y el $43.21 \%$ (figura 5). Además, representa entre 3 y 4 veces el $\mathrm{Al}$, el cual se ubica entre el $10.34 \%$ y el $12.35 \%$. De la misma forma, la MA representa más del doble de la MNA en los tres decretos.

Figura 5. Distribución de las RIR en relación con la formalidad del aprendizaje y el academicismo del repertorio

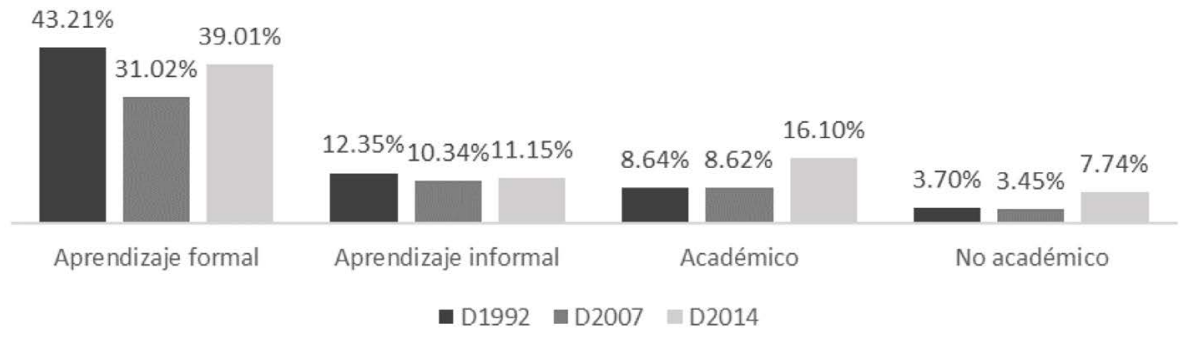


Dentro de las RIR relativas a la MA, la utilización de la palabra obra es la más frecuente en los tres decretos (figura 6). Aparecen en menor proporción prescripciones relativas a los instrumentos y agrupaciones académicas, a una actitud silenciosa frente a la audición musical y a la figura de la dirección. Con respecto a las RIR relacionadas con la MNA, se aprecia una tendencia hacia una mayor diversidad (figura 7). En este sentido, en el decreto D1992 solo hay unas pocas referencias a los instrumentos escolares, en el D2007 estas aumentan y se incorporan los folklórico-tradicionales y los propios de la MPU, y en el D2014 aumentan los primeros en más del doble, se mantienen estos últimos en porcentajes similares y aparecen débilmente las agrupaciones folklórico-tradicionales.

Figura 6. Distribución de las RIR relativa a la $M A$

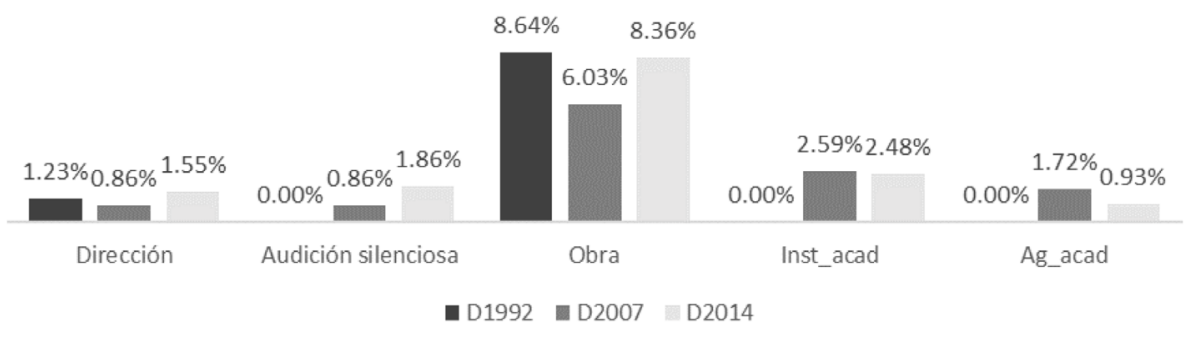

Figura 7. Distribución de las $R I R$ relativa a la $M N A$

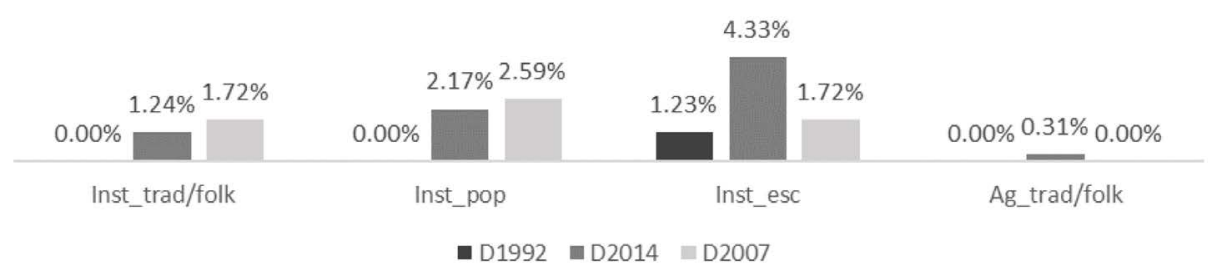

En cuanto a las RIR que hacen referencia al AF, el decreto D1992 se caracteriza por una mayor importancia de la teoría y análisis musical y de la notación escrita, el D2007 por realizar un gran énfasis en la palabra interpretación, y el D2014 por mostrar un mayor interés en la técnica, aunque manteniendo altos niveles de teoría, análisis y notación (figura 8). Por último, las RIR al AI son mayores en el decreto D1992 que en los siguientes, siendo la improvisación más abundante en el primero, la composición en el segundo y estableciéndose una igualdad entre ambas en el tercero (figura 9). 
Figura 8. Distribución de las RIR relativa al $A F$

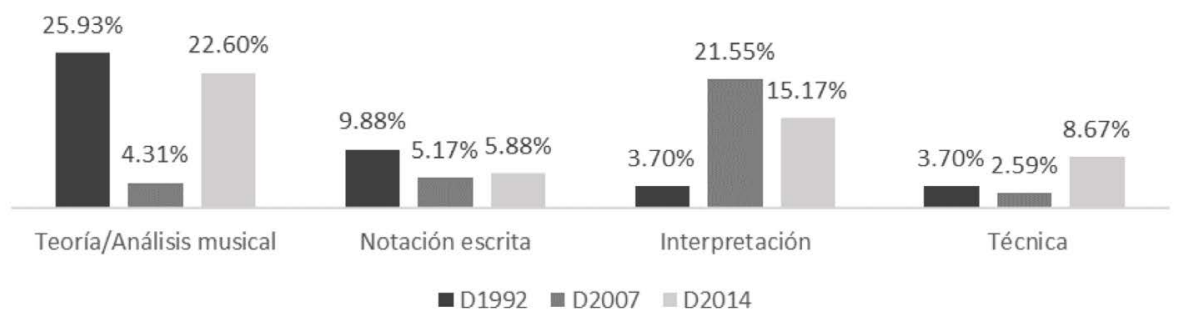

Figura 9. Distribución de las RIR relativa al AI

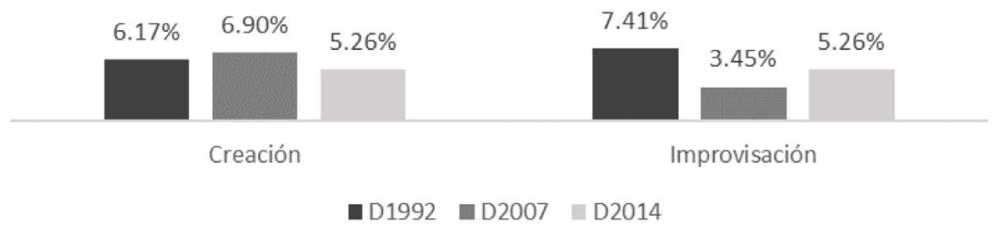

\section{Análisis cualitativo de las preferencias de los estudiantes y el repertorio académico}

Los resultados relativos al análisis cualitativo de las RDR y las RIR se muestra en dos apartados diferenciados: la presencia de las preferencias musicales de los estudiantes y el tratamiento que recibe el repertorio académico.

Las preferencias musicales de los estudiantes

Las referencias explícitas a las preferencias e identidades de los estudiantes son inexistentes en el decreto D2014. Solo pueden identificarse de forma indirecta y en ocasiones ambiguas algunas formulaciones que, sin necesariamente hacer mención a aquellas, sí podrían ser utilizadas como justificación para su uso educativo. Así, la introducción prescribe la utilización de "manifestaciones artísticas presentes y pasadas" y en un solo contenido de tercer curso se explicita el uso de "canciones de actualidad del entorno". Por otra parte, en los elementos curriculares de $5^{\circ}$ y $6^{\circ}$ curso se hace referencia a los instrumentos de la MPU y a los electrónicos, respectivamente. No obstante, el tratamiento que reciben estos es cualitativamente inferior al de otras familias instrumentales como la escolar, la orquestal y la folklórico-tradicional, ya que solo se prescribe su identificación visual, pero no auditiva, como si sucede en el resto.

De modo similar, el decreto D2007 no formula referencias explícitas a las preferencia e identidades musicales de los estudiantes, pero sí que aparecen menciones indirectas a las mismas. Por ejemplo, en la introducción se señala que la música cultural propia constituye "una memoria que corre el riesgo de perderse en una excesiva globalización de estéticas, productos y formas de vida" (Comunitat-Valenciana, 2007, p. 30296). Esta afirmación puede interpretarse como un rechazo a la 
MPU globalizada por generar un protagonismo que puede desplazar a la considerada como música propia, que podría identificarse con la tradicional valenciana. En la redacción de la contribución del área a las competencias y de los objetivos, este decreto también hace referencia al entorno próximo y la realidad cotidiana del alumnado, así como al tiempo presente, siendo elementos que pueden justificar la incorporación en el aula de las preferencias de los estudiantes. Finalmente, a nivel de contenidos y criterios de evaluación, aparece una referencia aislada a las canciones populares y otras relacionadas con el tiempo presente, el entorno y el medio social y cultural propio.

En el decreto D1992 tampoco aparece ninguna mención explícita a las preferencias e identidades musicales de los estudiantes. No obstante, sí que presenta un enfoque más constructivista que enfatiza en diversos momentos la importancia de los conocimientos previos y la realidad del alumnado como punto de partida para los aprendizajes posteriores.

\section{El repertorio académico}

Por lo que respecta al repertorio académico occidental, este presenta algunas referencias en el decreto D2014. Por ejemplo, en la introducción prescribe la utilización en el aula de "los grandes compositores universales". Por otro lado, en los contenidos del bloque de interpretación musical de los cursos $4^{\circ}$ y $5^{\circ}$ se hace mención al trabajo de "piezas clásicas". Sin embargo, lo más Ilamativo son las referencias a dicho repertorio a través de su clasificación en siglos históricos. En este sentido, a partir del tercer curso se observa en el repertorio prescrito una evolución cronológica que comienza en la música del período comprendido entre los siglos XVII y XVIII y va ampliando progresivamente sus márgenes. Esto se puede interpretar como una referencia a la música académica occidental, organizada tradicionalmente en siglos y articulada alrededor del período barroco y clásico, el cual se va expandiendo. Se introducen en el sexto curso otros géneros como el flamenco y el jazz, haciendo así alusión a música tradicional y popular urbana. Sin embargo, si bien la música académica occidental se vincula a actividades de audición y reconocimiento, estos dos últimos estilos se presentan asociados con actividades valorativas y de relación con el contexto.

Por otra parte, en el decreto D1992 no hay ninguna referencia al repertorio musical académico que presente interés de ser objeto del análisis cualitativo que complemente el cuantitativo ya presentado. Igualmente sucede con el decreto D2007, a excepción de una referencia a "la música universal" (Comunitat-Valenciana, 2007, p. 30296) en la introducción.

\section{Discusión y CONCLUSIONES}

En este estudio se ha analizado la evolución del repertorio musical prescrito en la Comunidad Valenciana en la etapa de educación primaria dentro del período democrático que ha tenido lugar durante las últimas tres décadas. Para ello, se ha realizado un análisis de contenido mixto. En primer lugar, se ha medido el grado de determinación de las referencias directas al repertorio. En este sentido, se ha 
encontrado que la cantidad de repertorio que viene explícitamente determinado por las prescripciones curriculares es escasa, habiendo un gran margen de indeterminación en el que los docentes pueden elegir qué músicas quieren utilizar en sus aulas. Esto implica que no se trata mayoritariamente de un currículum cerrado que establezca de forma unívoca los contenidos que se deben trabajar, sino que deja un margen de maniobra para que los docentes elijan qué piezas van a emplear. Por tanto, se hace necesario una formación del profesorado, tanto inicial como permanente, que les ayude a establecer criterios sobre el tipo de repertorio que quieren utilizar en el aula en función de los objetivos que se plantean dentro de la asignatura.

Con respecto a las referencias directas que aluden de forma determinada al género, estilo, o período musical, se ha observado un salto significativo a partir de 2007 hacia una mayor diversidad. No obstante, los dos repertorios protagonistas en la actualidad son la música folklórico-tradicional y la académica, que experimenta un aumento progresivo a lo largo de los tres decretos. Esto se puede complementar con el análisis sobre las referencias indirectas, en el que se ha encontrado que el aprendizaje formal y la música académica tienen una presencia muy superior a la del aprendizaje informal y la música no académica. Además, la música popular urbana recibe en ocasiones un trato de inferioridad.

Estos resultados son consistentes con otros trabajos que han observado también una hegemonía indirecta de la música académica (Cain, 2015; Economidou, 2006; Green, 2009; Hess, 2015). La mayoría señalan que, a pesar de que las reformas del currículum musical prescriban la inclusión de una mayor de diversidad musical y un enfoque multicultural, el protagonismo de la notación escrita, de conceptos teóricos como el metro, las dinámicas o articulaciones, y de destrezas propias de la música académica, hacen que músicas como la popular urbana u otras de tradiciones no occidentales queden excluidas del mismo. Frente a esto, sería interesante incluir en el currículum oficial las estrategias de aprendizaje propias de algunos géneros no académicos, fomentando la escucha y la oralidad, la toma de decisiones en pequeños grupos colaborativos y los espacios para la improvisación (Chua y Ho, 2017; Davis, 2013; Green, 2004, 2006; Snell, 2009; Wright, 2008).

En cuanto al análisis cualitativo, se ha encontrado que en las introducciones del área de educación artística de los últimos dos decretos se alude a la necesidad de introducir en el aula "la música universal" y a "los grandes compositores universales", organizando en la legislación actual la música académica en torno a los siglos XVII y XVIII. Estas referencias a la universalidad de la música académica occidental están estrechamente relacionadas con algunas ideas construidas entre finales de siglo XVIII y a lo largo del siguiente. Entre ellas se encuentran la autonomía de la música y su concepción de esta en tanto que manifestación autorreferente e independiente de todo contexto (Dahlhaus, 2006); la idea del genio compositor, que trasciende las tradiciones y reglas establecidas (Goehr, 1994; Samson, 2001b; Taruskin, 2005), y el canon académico occidental, que determina un conjunto de obras y compositores de excepcional valor y grandeza (Gloag, 2015; Samson, 2001a).

Sin embargo, desde el surgimiento de la nueva musicología en la década de 1980, esta concepción romántica de la música ha sido fuertemente criticada. Por un lado, 
la autonomía musical ha sido descartada por la inevitable relación que guarda con los condicionamientos estructurales del entorno cultural y social de producción y recepción (Citron, 2000; Goehr, 1994; Leppert y McClary, 1996; Small, 1989). Por el otro, la concepción de un canon occidental de validez universal se ha analizado retrospectivamente como una construcción histórica que legitima la ideología moral de grupos culturalmente dominantes (Citron, 2000; Goehr, 2002; Weber, 1992). Más aún, se ha visto como un instrumento de exclusión frente a la diversidad e incluso como una forma encubierta de colonialismo, imperialismo, sexismo, racismo y elitismo (Bohlman, 1992; Citron, 2000; Helm, 1994; Samson, 2001a).

Por tanto, se trata de una concepción de la música que, utilizada en el ámbito educativo, puede no ser respetuosa e inclusiva con respecto a la diversidad de manifestaciones e identidades culturales presentes en la sociedad en general y en los centros escolares en particular, a las cuales subordina. En este sentido, sería interesante implementar políticas educativas que enfocasen la educación musical primaria desde una perspectiva multicultural que trate de forma equitativa la diversidad musical (Campbell, 2018), ocupando la música académica un lugar no privilegiado y presentándose como un fenómeno situado histórica y geográficamente.

Por otro lado, no existe ninguna referencia específica a las preferencias musicales de los estudiantes en ninguno de los desarrollos curriculares estudiados, las cuales se corresponden con la música popular urbana (Cremades, Lorenzo, y Herrera, 2010; De Quadros y Quiles, 2010; de Vries, 2010; Dobrota y Ercegovac, 2019; Marín-Liébana et al., 2021). No obstante, sí se han encontrado referencias puntuales e indirectas que podrían justificar su utilización en el aula. A esto se suman las posibilidades didácticas que ofrecen los espacios de indeterminación curricular que, en este caso, si bien no las prescriben, sí que permiten que los docentes incorporen las preferencias de sus estudiantes. En este sentido, la perspectiva multicultural propuesta en el párrafo anterior no debería ceñirse a un currículum apriorístico, es decir, que establezca de antemano las manifestaciones musicales que deben formar parte del currículum. En su lugar, sería interesante que mantuviera una parte del repertorio abierta y estableciera la necesidad de dar voz al alumnado para que pudiera decidir qué tipo de música quiere trabajar en el aula (Flutter y Rudduck, 2004; Green, 2004; Laurence, 2009; Marín-Liébana et al., 2020b; Rimmer, 2013).

A la ausencia de referencias específicas a las preferencias de los estudiantes, se añade el tratamiento desigual que recibe la música popular urbana con respecto a otros géneros. Por ejemplo, se la asocia con actividades valorativas y de relación con el contexto, mientras que otros estilos como la música académica se prescriben a través de actividades de audición y reconocimiento, lo que contribuye a reforzar la idea de esta última como elemento formal, autónomo y superior. Además, en D2007 se puede encontrar un rechazo a la globalización musical por suponer una amenaza para la memoria de la cultura musical propia. Esto es congruente con algunos estudios que señalan el rechazo a la música popular urbana actual por ser considerada estéticamente inferior y moralmente dañina para los estudiantes (Boespflug, 2004; Hebert y Campbell, 2000; Humphreys, 2004).

Frente a esta situación, convendría incorporar en la formación docente reflexiones críticas sobre la construcción del gusto musical y la hegemonía del canon occidental 
(Weber, 1992), así como sobre la complejidad y dinamismo de la dimensión cultural de los fenómenos musicales (May, 2009). Es decir, sería interesante deconstruir la estética trascendental heredada de tradiciones como el pitagorismo, el platonismo, el cristianismo y el romanticismo (Fubini, 2005), que sitúa la música académica occidental en un espacio privilegiado, y transitar hacia una concepción del juicio estético en tanto que construcción subjetiva (Marín-Liébana et al., 2021). Desde esta perspectiva, deja de tener sentido el establecimiento de jerarquías estéticas absolutas, cobra importancia la comprensión de los significados y funciones de las diferentes manifestaciones musicales en sus respectivos contextos socioculturales, y se inicia un camino discursivo que podría facilitar la incorporación curricular de las preferencias musicales de los estudiantes.

A modo de síntesis, en este trabajo se ha comprobado cómo el repertorio musical viene determinado por el currículum oficial en diferentes niveles. Por un lado, las referencias directas al repertorio dejan importantes espacios de indeterminación en los que el docente puede elegir el tipo de música que quiere utilizar en su aula. Dentro de la determinación, el repertorio predominante es el de la música folklóricotradicional, seguido por la académica occidental en el decreto actual. Asimismo, las referencias indirectas favorecen a esta última y al aprendizaje formal, a lo que se añade el tratamiento de superioridad que recibe al ser considerada como música universal y al estar relacionada en algunos elementos curriculares con actividades de mayor importancia que otros estilos. Además, las referencias a las preferencias e identidades de los estudiantes son inexistentes en los tres decretos, y algunas de las pocas menciones que recibe la música popular urbana la sitúan en inferioridad con respecto a otros géneros.

Por tanto, aun a riesgo de simplificar en exceso un análisis que ha sido realizado a través de múltiples capas, se puede afirmar que el currículum musical prescrito para la Comunidad Valenciana durante el período democrático actual tiene un carácter academicista y no reconoce ni incorpora las preferencias musicales de los estudiantes. A partir de esto, se recomienda la incorporación en los sucesivos desarrollos curriculares de referencias explícitas a la importancia de la utilización curricular de dichas preferencias como una forma de reconocer sus identidades y ofrecerles un espacio de participación en el aula. Además, sería conveniente introducir en los programas de formación del profesorado reflexiones relativas a los criterios de selección del repertorio y a la deconstrucción de la estética trascendental, así como recursos y estrategias que favorezcan el aprendizaje informal.

Finalmente, en futuros estudios, sería interesante comprobar si lo observado en este trabajo sucede de la misma manera en otras comunidades autónomas. De igual forma, aportaría un gran valor conocer los procesos por los cuales estos decretos han sido redactados, para lo cual sería necesario desarrollar una investigación de corte cualitativo que entrevistase a los individuos que formaron parte en su elaboración. Igualmente, sería valioso indagar en las formas de recepción del currículum oficial por parte de los docentes y la lectura que del mismo hacen las editoriales y los autores de los manuales escolares a la hora de diseñarlos. Todo esto contribuiría a mejorar la comprensión que se tiene sobre la elaboración e implementación de las políticas curriculares en materia de educación musical y a orientar futuras reformas legislativas. 


\section{REFERENCIAS BIBLIOGRÁFICAS}

Abrahams, F. (2007). Musicing Paulo Freire: a critical pedagogy for music education. En P. McLaren y J. L. Kincheloe (Eds.), Critical Pedagogy: Where Are We Now? (pp. 223-237). Nueva York: Peter Lang.

Apple, M., Au, W. y Gandin, L. A. (2009). Mapping Critical Education. En M. Apple, W. Au, y L. A. Gandin (Eds.), The Routledge International Handbook of Critical Education (pp. 3-19). Nueva York: Routledge.

Barghi, R., Zakaria, Z., Hamzah, A. y Hashim, N. H. (2017). Heritage education in the Primary School Standard Curriculum of Malaysia. Teaching and Teacher Education, 61, 124-131. https://doi.org/10.1016/j.tate.2016.10.012

Bohlman, P. V. (1992). Epilogue: Musics and Canons. En K. Bergeron y P. V Bohlman (Eds.), Disciplining Music (p. 210). Chicago: The University of Chicago Press.

Bresler, L. (2000). The Relationship of School Art, National Goals, and Multilayered Cultures. Arts Education Policy Review, 101(5), 3-8. https://doi. org/10.1080/10632910009600266

Cain, M. (2015). Musics of 'The Other': Creating musical identities and overcoming cultural boundaries in Australian music education. British Journal of Music Education, 32(1), 71-86. https://doi.org/10.1017/S0265051714000394

Campbell, P. S. (2018). Music, Education, and Diversity. Nueva York, Londres: Teachers College.

Catling, S. (2014). Giving younger children voice in primary geography: empowering pedagogy - a personal perspective. International Research in Geographical and Environmental Education, 23(4), 350-372. https://doi.org/10.1080/10382046.20 14.946321

Chua, S. L. y Ho, H. P. (2017). Towards 21 st-century music teaching-learning. Reflections on student-centric pedagogic practices involving popular music in Singapore. En G. D. Smith, Z. Moir, M. Brennan, S. Rambarran y P. Kirkman (Eds.), The Routledge Research Companion to Popular Music Education. Routledge.

Citron, M. J. (2000). Gender and the Musical Canon. Urbana: University of Illinois Press.

Comunitat-Valenciana. DECRETO 111/2007, de 20 de julio, del Consell, por el que se establece el currículo de la Educación Primaria en la Comunitat Valenciana (2007). España.

Cremades, R., Lorenzo, O. y Herrera, L. (2010). Musical tastes of secondary school students' with different cultural background: a study in the Spanish north African city of Melilla. Musicae Scientiae, XIV(1), 121-141. https://doi. org/10.1177/102986491001400105

Davis, S. G. (2013). Informal learning processes in an elementary music classroom. Bulletin of the Council for Research in Music Education, 198, 23-50. https://doi. org/10.5406/bulcouresmusedu.198.0023

De Quadros, J. F. S., y Quiles, O. L. (2010). Musical Preferences of High School Students in Brazil: the Case of Vitória, Espírito Santo. Musica Hodie, 10(1), 109-128. https://doi.org/10.5216/mh.v10i1.12829 
de Vries, P. (2010). What we want: the music preferences of upper primary school students and the ways they engage with music. Australian Journal of Music Education, 1, 3-16.

Dobrota, S. y Ercegovac, I. R. (2019). Adolescent's musical preferences with regard to some socio-demographic variables. Odgojne znanosti, 11(2), 381-398.

Economidou, N. (2006). Reflecting on the Curriculum: The Case of the Cyprus Music Curriculum for Primary Education. Arts Education Policy Review, 107(4), 31-38. https://doi.org/10.3200/AEPR.107.4.31-38

Flutter, J. y Rudduck, J. (2004). Consulting Pupils: What's in It for Schools. Nueva York: Routledge Falmet.

Fubini, E. (2005). La estética musical desde la Antigüedad hasta el siglo XX. Madrid: Alianza.

Gay, G. (2000). Culturally Responsive Teaching. Theory, Research and Practice. Nueva York: Teachers College.

Giroux, H. (1989). Schooling as a Form of Cultural Politics: Towards a Pedagogy of and for Difference. En H. Giroux y P. McLaren (Eds.), Critical Pedagogy, the State, and Cultural Struggle (pp. 125-151). Nueva York: State University of New York Press.

Gloag, K. (2015). "A Thing of the Past": Canon Formation and the Postmodern Condition. En V. Kurkela y M. Mantere (Eds.), Critical Music Historiography: Probing Canons, Ideologies and Institutions (pp. 227-237). Surrey: Ashgate. https://doi. org/10.4324/9781315575049

Goehr, L. (1994). The imaginary museum of musical works. New York: Oxford University Press.

Goehr, L. (2002). In the shadow of the Canon. Musical Quarterly, 86(2), 307-328. https://doi.org/10.1093/musqtl/gdg012

Gowan, J. (2016). A Polysemy of Meanings : Music Education for Critical Pedagogy. Canadian Music Educator, 57(3), 23-28.

Green, L. (2004). What Can Music Educators Learn from Popular Musicians? En C. X. Rodríguez (Ed.), Bridging the Gap. Popular Music and Music Education (pp. 225247). Reston: MENC: The National Association for Music Education.

Green, L. (2005). The Music Curriculum as Lived Experience: Children's "Natural" Music-Learning Processes. Music Educators Journal, 91(4), 27-32. https://doi. org/10.2307/3400155

Green, L. (2006). Popular music education in and for itself, and for "other» music: Current research in the classroom. International Journal of Music Education, 24(2), 101-118. https://doi.org/10.1177/0255761406065471

Green, L. (2009). Significado musical y reproducción social: defensa de la recuperación de la autonomía. En D. K. Lines (Ed.), La educación musical para el nuevo milenio. Madrid: Morata.

Green, L. (2011). The globalization and Localization of Learning, teaching, and Musical Identity. En L. Green (Ed.), Learning, Teaching, and Musical Identity: Voices across cultures (pp. 1-19). Bloomington: Indiana University Press. 
Grembis, H. y Schellberg, G. (2003). Musical preferences of elementary school children. En R. Kopiez, A. C. Lehmann, I. Wolther y C. Wolf (Eds.), Abstracts of the 5th triennial conference of the European Society for the Cognitive Science of Music ( $p$. 324). Hannover: University of Hannover. https://doi.org/10.2307/3344766

Gurgel, R. (2019). The Tanglewood Symposium: Popular Music Pedagogy from 1967 to Today. Music Educators Journal, 105(3), 60-65. https://doi. org/10.1177/0027432119831752

Hargreaves, D. J., MacDonald, R. y Miell, D. (2017). The changing identity of musical identities. En R. MacDonald, D. J. Hargreaves y D. Miell (Eds.), Handbook of musical identities (pp. 3-23). New York: Oxford University Press.

Hargreaves, D. J., North, A. C. y Tarrant, M. (2010). Musical preference and taste in childhood and adolescence. En G. E. McPherson (Ed.), The child as musician (pp. 135-154). New York: Oxford University Press. https://doi.org/10.1093/acprof: oso/9780198530329.003.0007

Helm, E. E. (1994). The canon and the curricula. A Study of Musicology and Ethnomusicology Programs in America. Stuyvesant: Pendragon Press.

Hess, J. (2015). Decolonizing music education: Moving beyond tokenism. International Journal of Music Education, 33(3), 336-347. https://doi. org/10.1177/0255761415581283

Ishii, Y. (2018). The roles played by a common language and music education in modernization an nation-state building in Asia. Espacio, Tiempo y Educación, 5(2), 55-76. https://doi.org/10.14516/ete.221

Kelly, D. M., Pomerantz, S. y Currie, D. (2005). Skater girlhood and emphasized femininity: "you can't land an ollie properly in heels". Gender and Education, 17(3), 229-248. https://doi.org/10.1080/09540250500145163

Krippendorff, K. (2013). Content analysis: an introduction to its methodology. Thousand Oaks: SAGE.

Künzler, D. (2011). South African rap music, counter discourses, identity, and commodification beyond the prophets of Da City. Journal of Southern African Studies, 37(1), 27-43. https://doi.org/10.1080/03057070.2011.552540

Lamont, A., Hargreaves, D. J., Marshall, N. A. y Tarrant, M. (2003). Young people's music in and out of school. British Journal of Music Education, 20(3), 229-241. https://doi.org/10.1017/S0265051703005412

Larson, R. y Kubey, R. (1983). Television and music: contrasting media in adolescent life. Youth and Society, 15(1), 13-31. https://doi.org/10.1177/0044118X83015001002

Laurence, F. (2009). Listen to Children: Voice, Agency and Ownership in School Musicking. En R. Wright (Ed.), Sociology and Music Education (pp. 243-262). Farnham: Ashgate.

Leppert, R. y McClary, S. (Eds.). (1996). Music and Society. The Politics of Composition, Performance and Reception. Nueva York: Cambridge University Press.

Lucas, G., Ricardo, L., Queiroz, S., Prass, L., Ribeiro, F. H. y Aredes, R. D. O. (2016). Afro-Brazilian Musical Cultures: Perspectives for Educational Conceptions and Practices in Music. The World of Music, 5(1), 135-158. 
Lum, C. H. y Dairianathan, E. (2014). Mapping musical learning: An evaluation of research in music education in Singapore. International Journal of Music Education, 32(3), 278-295. https://doi.org/10.1177/0255761413491206

Marín-Liébana, P. y Botella, A. M. (2020). Las preferencias musicales de los estudiantes de $5^{\circ}$ y $6^{\circ}$ de Educación Primaria . Una oportunidad para implementar un enfoque de tipo sociocrítico. Revista Internacional de Educación Musical, 8, 15-24. https://doi.org/10.1177/2307484120956512

Marín-Liébana, P., Blasco, J. S. y Botella, A. M. (2020a). Hacia una comprensión de las identidades musicales de los estudiantes. Desde los primeros años hasta la adolescencia. ENSAYOS, Revista de la Facultad de Educación de Albacete, 35(2), 87-102.

Marín-Liébana, P., Blasco, J. S. y Botella, A. M. (2020b). La utilización de las preferencias musicales de los estudiantes desde un enfoque crítico. Hacia una educación musical democratizadora. Opus, 26(3), 1-24. https://doi.org/10.20504/ OPUS2020C2613

Marín-Liébana, P., Blasco, J. S. y Botella, A. M. (2021). Las preferencias musicales de los estudiantes: hacia un reconocimiento de sus identidades culturales. Vivat Academia, 154, 1-22.

May, S. (2009). Critical multiculturalism and education. En J. A. Banks (Ed.), The Routledge International Companion to Multicultural Education (pp. 33-48). New York and London: Routledge. https://doi.org/10.4324/9780203881514

Megías, I. y Rodríguez, E. (2003). Jóvenes entre sonidos. Madrid: Fundación de Ayuda contra la Drogadicción.

Monk, M.yPoston, M. (1999).AComparison ofMusic and ScienceEducation. Cambridge Journal of Education, 29(1), 93-101. https://doi.org/10.1080/0305764990290107

Poblete, C. (2010). Enseñanza musical en Chile: continuidades y cambios en tres reformas curriculares (1965, 1981, 1996-1998). Revista Musical Chilena, 214, 12 35. https://doi.org/10.4067/S0716-27902010000200004

Pugach, M. C., Gomez-Najarro, J. y Matewos, A. M. (2018). A Review of Identity in Research on Social Justice in Teacher Education: What Role for Intersectionality? Journal of Teacher Education, 00(0), 1-13. https://doi.org/10.1177/0022487118760567

Rimmer, M. (2013). Can you hear me now? Musical values, education and voice. Media International Australia incorporating Culture and Policy, 148, 135-144. https:// doi.org/10.1177/1329878X1314800115

Samson, J. (2001a). Canon. En Grove Music Online (pp. 1-3). Oxford University Press.

Samson, J. (2001b). The great composer. En J. Samson (Ed.), The Cambridge History of Nineteenth-Century Music (pp. 259-284). Cambridge: Cambridge University Press.

Small, C. (1989). Música. Sociedad. Educación. Madrid: Alianza Editorial.

Snell, K. (2009). Embodied performance in popular music: Considerations for music education through an examination of the Dresden dolls. Journal of Popular Music Studies, 21(1), 59-75. https://doi.org/10.1111/j.1533-1598.2009.01169.X

Taruskin, R. (2005). The Oxford History of Western Music. Oxford: Oxford University Press. 
Weber, W. (1992). The Rise of Musicl Classics in Eighteenth-Century England. A Study in Canon, Ritual, and Ideology. Oxford: Clarendon Press.

Westerlund, H., Partti, H. y Karlslen, S. (2017). Identity formation and agency in the diverse music classroom. En R. MacDonald, D. J. Hargreaves y D. Miell (Eds.), Handbook of musical identities (pp. 493-509). New York: Oxford University Press. https://doi.org/10.1093/oxfordhb/9780198722946.013.45

Woody, R. (2007). Popular Music in Schools: Remixing the Issues. Music Educators Journal, 93(4), 32-37. https://doi.org/10.1177/002743210709300415

Wright, R. (2008). Kicking the habitus: power, culture and pedagogy in the secondary school music curriculum. Music Education Research, 10(3), 389-402. https://doi. org/10.1080/14613800802280134

Wright, R. (2009). Democracy, Social Exclusion and Music Education: Possibilities for Change. En R. Wright (Ed.), Sociology and Music Education (pp. 263-281). Farnham: Ashgate. 